\title{
Flexible and stable optical parametric oscillator based laser system for coherent anti-Stokes Raman scattering microscopy
}

\author{
Wei Zhang ${ }^{1}$, Maddy Parsons ${ }^{2}$ and Gail McConnell ${ }^{1}$ \\ ${ }^{1}$ Centre for Biophotonics, SIPBS, University of Strathclyde, Glasgow G4 0NR, United Kingdom \\ ${ }^{2}$ Randall Division of Cell and Molecular Biophysics, King's College London, London, United Kingdom \\ Email: wei.zhang.100@strath.ac.uk \\ Telephone: +44-(0)141-5484738
}

\begin{abstract}
The characteristics of a stable and flexible laser system based on a synchronously pumped optical parametric oscillator (OPO) is presented. This OPO can offer very stable operation with both $\sim 1$ ps and $\sim 300$ fs outputs over a broad wavelength range, i.e. $920 \mathrm{~nm}$ to $1200 \mathrm{~nm}$. Combining the pump tuning with the OPO tuning, a total Raman range of $1900 \mathrm{~cm}^{-1}$ to $5500 \mathrm{~cm}^{-1}$ is accessible. For maximum spectral sensitivity, the CARS microsope based on the ps laser system is presented in detail. The lateral resolution of the microscope is diffraction limited to be about 390nm. Fast wavelength switching (sub-second) between two Raman vibrational frequencies, i.e. $2848 \mathrm{~cm}^{-1}$ for $\mathrm{C}-\mathrm{H}$ aliphalic vibrations and $3035 \mathrm{~cm}^{-1}$ for $\mathrm{C}-\mathrm{H}$ aromatic vibrations is presented as an example, although this also extends to other Raman frequencies. The possibility of obtaining a multimodal imaging system based on the fs laser system is also discussed.
\end{abstract}




\section{Introduction}

Coherent anti-Stokes Raman scattering (CARS) microscopy based on the intrinsic vibrational signatures of molecules is a non-invasive imaging technique with chemical selectivity. CARS microscopy is a nonlinear process requiring at least two laser fields with frequencies of $\omega_{1}$ and $\omega_{2}$. When the beat frequency $\omega_{1}-\omega_{2}$ is tuned to match one of the vibrational resonances of the sample, a strong coherent anti-Stokes signal is generated which can be collected and used as the image contrast mechanism. It has been demonstrated to offer excellent 3D spatial resolution and high sensitivity (Chen et al., 2004). The common use of near infrared laser sources reduces the Rayleigh scattering and hence increases the penetration depth and makes it also very useful for in vivo imaging (Evans et al., 2005) given the low photo-toxicity at this spectral window.

Since the first demonstration of 3D CARS imaging with near infrared excitation and collinear beam geometry, CARS microscopy has been extensively studied and widely applied for many different fields (Evan et al., 2008). Clearly, these advances in CARS microscopy owe much to the developments of suitable and robust laser sources. The first generation of laser systems used for CARS microscopy were based on low repetition rate high pulse energy optical parametric amplifiers (Zumbusch et al., 1999, Hashimoto et al., 2000). These systems were very complex and difficult to use. Moreover, the low repetition rate and broad spectral bandwidth significantly limited the imaging speed and the spectral resolution of the system. A few years later in 2002, the development of the synchronization system in JILA (Jones et al., 2002) made it possible to synchronize two ps-pulsed Ti:sapphire oscillators with a timing jitter of around 20fs. The CARS microscope incorporating this laser system demonstrated broad frequency coverage together with excellent spatial and spectral resolution (Potma et al., 2002). However, this system was again very complex and the total cost was very high. Its synchronization system was also vulnerable to external perturbations which would lead to the deterioration of image quality. Similar laser systems involving synchronizing a ps-Tisapphire and a fs-Tisapphire had also been 
used for multiplex CARS spectroscopy (Volkmer, 2005) but the problems concerning cost and reliability remained.

Due to their inherent synchronization and broad spectral tunability, high repetition rate synchronously pumped optical parametric oscillator (OPO) systems have shown great suitability for CARS microscopy. The first system used for CARS imaging was an intra-cavity frequency doubled OPO based on a periodically poled potassium titanyl phosphate (PP-KTP) crystal (Evans et al., 2005). The OPO was synchronously pumped by a $10 \mathrm{~W}$ Nd: $\mathrm{YVO}_{4}$ laser at $1064 \mathrm{~nm}$. The frequency doubled signal beam covering $780 \mathrm{~nm}$ to $930 \mathrm{~nm}$ together with a portion of the $\mathrm{Nd}$ : $\mathrm{YVO}_{4}$ beam were used as the pump beam and the Stokes beam for CARS microscopy. This approach had been used for demonstration of high sensitivity video-rate in vivo imaging. However, the intra-cavity frequency doubling adds to the complexity of the system and significantly reduces the tolerance of cavity misalignment or cavity length detuning. Furthermore, the beam quality of the laser was found to be poor. In 2006, a broadly tuable dual-wavelength OPO system based on PP-KTP crystal was developed and applied to CARS imaging (Ganikhanov et al., 2006). The OPO was pumped by a frequency doubled mode locked $\mathrm{Nd}: \mathrm{YVO}_{4}$ laser, with the OPO designed to be singly resonant within the idler wavelength region. The generated idler waves together with the dissipated signal wave from the OPO were firstly overlapped and then used as the pump and the Stokes beams. The CARS microscope based on this system had shown many advantages over that using two synchronised Ti:Sapphire lasers, e.g. good spatial and spectral resolution, excellent sample penetration depth. However, due to the use of longer excitation wavelengths $(900 \mathrm{~nm}-1200 \mathrm{~nm})$, the generated anti-Stokes signal was above $700 \mathrm{~nm}$ in which region the quantum efficiency of the most photomultiplier tubes drops sharply. Since the spectral tuning could only be achieved by varying the crystal temperature, the tuning process was very slow (at least tens of mins). This would not be convenient if different Raman resonances need to be investigated for the same sample in a short period of time. Furthermore, few laboratories have access to a $\mathrm{Nd}: \mathrm{YVO}_{4}$ laser source while many already employ Ti:Sapphire systems for alternative nonlinear imaging techniques such as 
multi-photon fluorescence microscopy or harmonic generation imaging. Additionally, frequency doubling of the laser platform represents an optical loss and the fundamental output of the Ti:Sapphire laser would provide a more efficient solution. As such, an efficient and stable platform system for CARS microscopy based upon a Ti:Sapphire laser would present opportunities for the wider life sciences community to embrace CARS microscopy. The Spectra Physics Inspire and APE OPO PP Automatic systems employ OPO technology for CARS imaging, however, there remain several short-comings. The Inspire system is only designed to generate fs pulses and thus is very suitable for many applications such as multiphoton microscopy but the spectral bandwidth is too broad for efficient CARS imaging with high chemical specificity. Alternatively, the APE OPO offers the option of either ps or fs output but the signal wavelength of the OPO is typically longer than $1100 \mathrm{~nm}$. At these long wavelengths, the optical efficiency of commercial microscopes and objective lenses drops sharply [xxx]. Furthermore, fast wavelength switching is generally difficult to perform using these expensive systems.

In this paper, we present a flexible and stable OPO system synchronously pumped by a Ti:sapphire laser which can support both ps and fs pulsed operation in a broad spectral range of $920 \mathrm{~nm}$ to $1200 \mathrm{~nm}$. A CARS microsope based on the ps laser system is evaluated in detail along with a stability analysis of the laser imaging platform. Finally, a potential multimodal imaging system based on the fs laser system is proposed.

\section{Materials and Methods}

\section{a) Laser source-optical parametric oscillator}

Figure 1 shows the schematic setup of the CARS system. After passing through the beam splitter, $90 \%$ of the Ti:Sapphire pulse train (Coherent Mira Dual), operating at a $76 \mathrm{MHz}$ repetition rate, was used to pump an OPO. The Ti:Sapphire laser could generate $1.2 \mathrm{ps}$ and 200 fs pulses in the ps-configuration and fs-configuration, respectively. The OPO was based on a periodically poled lithium niobate (PPLN) crystal. The $0.5 \mathrm{~mm}$ thick, $10 \mathrm{~mm}$ long crystal had twelve separate domain-inverted 
gratings with periods of $17.6 \mu \mathrm{m}$ to $19.8 \mu \mathrm{m}$ for first-order quasi-phase matching (QPM) [Myers et al., 1995], suitable for pumping around $750 \mathrm{~nm}$, with signal and idler wavelengths in $0.91 \mu \mathrm{m}$ to $1.25 \mu \mathrm{m}$ and $1.87 \mu \mathrm{m}$ to $4.22 \mu \mathrm{m}$. The crystal was antireflection coated at both the pump wavelength and the calculated and hence anticipated signal wavelength tuning range of $920 \mathrm{~nm}$ to $1200 \mathrm{~nm}$. It was mounted in an oven to allow operation at elevated temperatures of up to $200{ }^{\circ} \mathrm{C}$ with a stability of $\pm 0.1{ }^{\circ} \mathrm{C}$. The bow-tie cavity consisted of four $\mathrm{CaF}_{2}$ cavity mirrors (Laseroptik $\mathrm{GmbH}$ ), i.e. two highly reflective (HR) curved mirrors with a radius of curvature of $100 \mathrm{~mm}$, one plane HR end mirror and one output coupling (OC) mirror. The mirror coatings were optimised for singly resonant operation across the signal wavelength tuning range. This cavity design resulted in a laser mode size of $16 \mu \mathrm{m}$ in radius in the crystal. The pump beam was focused into the crystal to a spot size of $\sim 13 \mu \mathrm{m}$ in radius in order to fulfill the optimal focusing conditions for parametric waves (Boyd et al., 1968; Guha et al., 1998). The combination of a half waveplate and a polarizer were employed as a variable attenuator for the incident pump power. As shown in Figure 1, $\sim 10 \%$ of the total pump power available from the Ti:sapphire was used as the pump beam in CARS process. This was picked off by the beam splitter and guided through an optical delay line to enable overlapping spatially and temporally with the OPO signal beam. The OPO signal beam was used as the Stokes beam in the CARS processs. The beams were combined at the dichroic mirror (z850dcspxr, Chroma Technology). Both laser beams were then focused onto an ADP crystal (type I, theta $=43.0^{\circ}$, United Crystals) with a size of $5 \mathrm{~mm}$ (length) $\times 5 \mathrm{~mm}$ (width) $\times 2 \mathrm{~mm}$ (thickness). The temporal overlapping between the two pulses was optimised by improving sum frequency mixing signal detected by a photomultiplier tube (PMT) (1P28 Hamamatsu Photonics).

With 1.2 ps pulse pumping, an output coupling mirror with $40 \%$ reflectivity was chosen to maximise the average output power of the OPO across the signal wavelength tuning range. A maximum output power of $210 \mathrm{~mW}$ was measured at a wavelength $1005 \mathrm{~nm}$, as shown in Figure 2. This was measured after a $785 \mathrm{~nm}$ 
long-wave pass filter (BLP01-785R-25, Semrock) with a transmission of $>97 \%$ across a range of $812 \mathrm{~nm}$ to $1200 \mathrm{~nm}$ and was obtained with a pump power of $\sim 880$ $\mathrm{mW}$ at $752 \mathrm{~nm}$. The oven temperature was set to be $393 \mathrm{~K}$ and the grating period was $19.0 \mu \mathrm{m}$. The pump depletion was measured to be above $80 \%$ and the typical slope efficiency for the signal wavelength output was $30 \%$. The amplitude stability of the OPO was also measured and compared with that of the pump laser, as shown in the inset of the Figure 2. For short periods (over 5 seconds), the OPO was found to be as stable as the Ti:Sapphire laser with amplitude stability of $0.9 \% \mathrm{rms}$. The stability of the OPO over 33 min was about $1.4 \%$ rms which was still comparable to Ti:Sapphire stability $(0.96 \% \mathrm{rms})$. The far field transverse mode of the signal beam was found to be $\mathrm{TEM}_{00}$ mode and the $\mathrm{M}^{2}$ factor of the beam profile was measured to be 1.05 across the entire signal wavelength tuning range.

In a first-order QPM device, the phase-matching condition can be described as

$$
\Delta k_{Q P M}=k_{p}-k_{s}-k_{i}-k_{g},
$$

where $k_{p}, k_{s}, k_{i}$ refer to the pump, signal and idler wave vectors; while $k_{g}=2 \pi / \Lambda$ is the grating vector of the phase-matched Fourier component of the modulated nonlinear susceptibility and $\Lambda$ is the period of the modulation assuming that all wave vectors are collinear with the grating vector [Fejer et al., 1992]. Tuning can be achieved by varying the pump wavelength, the crystal oven temperature, crystal grating period and cavity length. The pump tuning characteristics are summarised in the Table 1. At a temperature of $393 \mathrm{~K}$, each of 12 gratings was tested by scanning the pump wavelength at a step of $\sim 5 \mathrm{~nm}$. The signal wavelength could be tuned from about 920 $\mathrm{nm}$ to about $1200 \mathrm{~nm}$ for all the grating periods with different pump ranges. This tuning range was limited by the coatings of the cavity mirrors. The corresponding Raman range at $393 \mathrm{~K}$ was from $2375 \mathrm{~cm}^{-1}$ to $5365 \mathrm{~cm}^{-1}$.

In order to study the other tuning characteristics, the pump wavelength was selected to be $752 \mathrm{~nm}$. Figure 3 shows the theoretical tuning curves, calculated by using the freely-available nonlinear optical modelling software SNLO, and the measured values for both temperature and grating tuning. At each grating (only $18.2 \mu \mathrm{m}-19.8 \mu \mathrm{m}$ 
gratings were tested), a continous tuning range of $\sim 100 \mathrm{~nm}$ was obtained by varying the temperature from $323 \mathrm{~K}$ to $453 \mathrm{~K}$ and this corresponded to a Raman range of $\sim 1000 \mathrm{~cm}^{-1}$. At each temperature, grating tuning gave a wavelength increment of $\sim 20$ $\mathrm{nm}$ or a Raman shift of $\sim 200 \mathrm{~cm}^{-1}$. The grating tuning would be extremely useful for fast frequency tuning in the $\mathrm{C}-\mathrm{H}$ bonds region as the Raman shift between the peak of the $\mathrm{C}-\mathrm{H}$ aliphatic bond and the peak of the $\mathrm{C}-\mathrm{H}$ aromatic bond is around $200 \mathrm{~cm}^{-1}$. Combining the pump tuning with temperature and grating tuning, a total Raman range of $\sim 1900 \mathrm{~cm}^{-1}$ to $\sim 5700 \mathrm{~cm}^{-1}$ was therefore accessible by using this laser system. It shoud be noted that the laser system could not directly access the Raman finger print region $\left(<1900 \mathrm{~cm}^{-1}\right)$. However, it covers the $\mathrm{C}-\mathrm{H}$ bond region around $3000 \mathrm{~cm}^{-1}$ and therefore is very well suited for imaging lipids in biological samples which has been found to be the most successful application of the CARS microscopy [Evan et al., 2008].

All the measurements mentioned above were obtained after optimising the cavity length to achieve maximum power. However, due to the dynamics of the parametric gain, cavity length tuning could also result in the frequency shift of the signal wave and the change of the intensity profiles (Cheung et al., 1990; Hache et al., 1995). With a grating period of $19.2 \mu \mathrm{m}$, a pump wavelength of $752 \mathrm{~nm}$ and a temperature of 413 $\mathrm{K}$, the pulse duration at the maximum output was about $1 \mathrm{ps}$, assuming a sech ${ }^{2}$ pulse shape. The corresponding FWHM spectral width was measured to be about $8 \mathrm{~nm}$. This indicated the pulse at the maximum output power was strongly chirped. However, by shortening or lengthening the cavity, the spectral bandwidth could be significantly reduced. For example, the FWHM spectral width under a negative cavity detuning of $40 \mu \mathrm{m}$ was $1.9 \mathrm{~nm}$ or $\sim 19 \mathrm{~cm}^{-1}$ while the pulse duration was about $0.8 \mathrm{ps}$, as shown in Figure 4(a) and Figure 4(b). Here, the minimum spectral bandwidth of the OPO was limited by the pump spectral bandwidth. Hence the time-bandwidth product was about 0.4, shown in Figure 4(d), indicating a nearly transform limited pulse. The rather small amount of the chirp is still in a favourable range to achieve high contrast CARS image [Chen et al., 2004]. As shown in Figure 4(c), cavity length detuning in the 
range of $-40 \mu \mathrm{m}$ to $+10 \mu \mathrm{m}$, resulted in a fast continuous spectral shift of $\sim 100 \mathrm{~cm}^{-1}$ while the variation of the output power was less than $30 \%$.

\section{b) fs configuration}

With 200 fs pulse pumping, the tuning characteristics were similar to those observed with ps pulse pumping. Here, for brevity we only present data measured with the grating period of $19.2 \mu \mathrm{m}$. The maximum output power of $90 \mathrm{~mW}$ was obtained when the incident pump power was $620 \mathrm{~mW}$ at $752 \mathrm{~nm}$ and the oven temperature was set at $413 \mathrm{~K}$. The $50 \%$ reflectivity output coupler was used for this optimal output. The use of higher OC reflectivity was to compensate the less parametric gain of the fs OPO, due to the fact that the crystal length was long enough to cause significant temporal walk-off which was induced by the group velocity mismatch between the pump pulse and the signal pulse. The laser was therefore less efficient and the pump depletion was measured to be $57 \%$. The dependence of the pulse profiles and the spectra on the cavity length detuning was also investigated. As shown in Figure 5(a), the FWHM autocorrelation trace varied from 375 fs to 700 fs which corresponded to $\operatorname{sech}^{2}$ pulse duration of 213 fs to 398 fs as the cavity was detuned from $-60 \mu \mathrm{m}$ to $+50 \mu \mathrm{m}$. The peak wavelength shifted continuously from $940 \mathrm{~nm}$ to $1005 \mathrm{~nm}$. In the detuning region of $-10 \mu \mathrm{m}$ to $+30 \mu \mathrm{m}$, the pulse was chirped with a time-bandwidth product of $\geq 1$, as shown in Figure 5(b). Outside that region, the value reduced to $\sim 0.7 \quad(\sim 2.2$ times of the transform limit). As per the picosecond temporal regime, the OPO delivered a good quality $\mathrm{TEM}_{00}$ transverse mode $\left(\mathrm{M}^{2}<1.1\right)$ across the entire signal wavelength tuning range.

In addition to the generation of the IR wavelengths, there were four visible wavelength outputs that were simultaneously generated as a consequence of inadvertent phase-matching in the poled crystal: $2 \omega_{\mathrm{p}}, 2 \omega_{\mathrm{s}}, \omega_{\mathrm{p}}+\omega_{\mathrm{s}}, \omega_{\mathrm{p}}+\omega_{\mathrm{i}}$. Of these processes, the SHG of the signal and the SFG between the signal and the pump were the most efficient, with average powers of $\sim 10 \mathrm{~mW}$. It is found that the SHG of the signal was in two main regions, i.e. blue region $(471.82 \mathrm{~nm}-489.42 \mathrm{~nm})$ through the 
fourth order QPM process and green region $(515.76 \mathrm{~nm}-534.90 \mathrm{~nm})$ through the third order QPM process. The SFG of the pump and the OPO signal through fourth order QPM process produced the blue light in the range of $419.17 \mathrm{~nm}$ to $433.8 \mathrm{~nm}$. It should be noted that these spectral lines were also observed in the picoseconds OPO described above.

This fs-OPO system covering a very broad spectral region is suitable for many different imaging applications. The OPO signal, in the range of $920 \mathrm{~nm}$ to $1200 \mathrm{~nm}$, together with part of the Ti:sapphire output (700 nm to $950 \mathrm{~nm}$ ) could be very useful for two photon excitation over a significant wide range of fluorophores, especially those far-red fluorescent proteins such as the recently developed "fruit" probes including mCherry, mPlum and mTomato (Shcherbo et al., 2009). Moreover, a multiplex CARS system could also be achieved by simply chirping one of the two laser pulses (Onorato et al., 2007). Besides the suitability for two-photon and CARS imaging, the blue and green visible outputs add another capability of confocal microscopy. Hence, this laser system offers a diverse yet stable and inexpensive for simultaneous confocal, two-photon and CARS microscopy in both the picosecond and femtosecond temporal domains.

\section{c) Microscope platform}

The CARS platform employed in this work was modified from an IR corrected Olympus FV300/IX71 laser scanning confocal microscope. Similar to the modifications in the reference (Moger et al., 2008), one of the internal PMTs (R3896 Hamamatsu Photonics) in the scanhead was taken out and was used for forward detection. The temporally and spatially overlapped pulse trains from the Ti:Sapphire and the OPO were coupled collinearly into the scanhead and focused on the same spot of the sample with a water-immersion objective (Olympus, UPLSAPO 60X, NA 1.2). A $700 \mathrm{~nm}$ long wave pass dichroic mirror (700dcxru-xt, Chroma Technology) was placed above the condenser lens $(\mathrm{NA}=0.55)$ to separate the CARS signal and the fundamental waves. The CARS signal was guided through a stack of band pass filters 
(three Chroma et620/60-2p filters and one Chroma et700sp-2p8 filter) and then focused into the PMT. The transmitted fundamental waves were detected by a fibre coupled PMT to form the transmission images. All the PMTs were synchronously controlled by the Olympus FluoView software.

\section{Results}

Polystyrene (PS) beads (Polysciences Europe $\mathrm{GmbH}$ ) were used to evaluate the CARS system. By fixing the pump wavelength at $752 \mathrm{~nm}$ while tuning the Stokes wavelength from the OPO in $2 \mathrm{~nm}$ steps from $952.5 \mathrm{~nm}$ to $980.5 \mathrm{~nm}$ (corresponding to Raman shifts from $2800 \mathrm{~cm}^{-1}$ to $3100 \mathrm{~cm}^{-1}$ ) a CARS spectrum of $1 \mu \mathrm{m}$ PS was obtained as shown in Figure 6(a). For comparison, the spontaneous Raman spectrum of the same PS beads was also recorded by using a micro-Raman system (inVia, Renishaw, UK). A slight red-shift of the CARS spectrum was induced from the interference between the resonant and the nonresonant CARS signals (Levenson and Kano, 1988). Since the spectral bandwidths for both pump and Stokes pulses were around $10 \mathrm{~cm}^{-1}$ and $19 \mathrm{~cm}^{-1}$ respectively, the spectral resolution given by the convolution of the spectral widths of the two exciting laser beams, was estimated to be $\sqrt{10^{2}+19^{2}}=21.5 \mathrm{~cm}^{-1}$ (Chen et al., 2001). The spectral resolution could be further improved to be around $14 \mathrm{~cm}^{-1}$ if a custom designed birefringent filter was inserted into the OPO cavity to narrow the spectral bandwidth of the Stokes pulse (Connor et al., 2004). Figure 6 (b) shows the lateral CARS image of the $1 \mu \mathrm{m}$ PS beads spin coated onto a coverslip. The pump power and Stokes power on the sample were approximately $20 \mathrm{~mW}$ and $40 \mathrm{~mW}$ respectively. This image (262 pixels x 165 pixels) was cropped from a full image with an original size of 512 pixels x 512 pixels taken at a frame rate of $1 \mathrm{~Hz}$. The image (95 pixels $\mathrm{x} 47$ pixels) on a single bead in the xz plane is also shown in Figure 6 (c). The lateral FWHM and the axial FWHM were found to be around $580 \mathrm{~nm}$ and $1.28 \mu \mathrm{m}$, respectively, as shown in Figure 6 (d) and Figure 6 (e). The lateral FWHM width is much narrower than the actual size of the bead. This can be explained by the fact that the CARS signal is the square module of 
the coherent summation of the CARS radiation fields and thus the conventional method, based on the convolution of the object with the point spread function of the excitation/detection profile, cannot be used (Chen et al. 2002a). The axial resolution is thus determined to be $1.28 \mu \mathrm{m}$. The lateral resolution of the CARS microscope was evaluated to be about $390 \mathrm{~nm}$ by using $200 \mathrm{~nm}$ PS beads which was very close to the theoretical value of $\sim 380 \mathrm{~nm}$ calculated by using the method of Cheng et al. (Chen et al. 2002b).

As previously mentioned, fast wavelength switching of the Stokes beam can be performed by tuning the OPO crystal grating. This enables the possibility to rapidly monitor different Raman active bands within the same sample. To demonstrate this capability, we imaged a mixture of $10 \mu \mathrm{m}$ PS beads and $1.4 \mu \mathrm{m}$ poly-methylmethacrylate (PMMA) beads. Figure 7 shows the CARS images taken at two different Raman shifts, i.e. $\sim 2848 \mathrm{~cm}^{-1}$ and $\sim 3035 \mathrm{~cm}^{-1}$. Since PS beads have both $\mathrm{C}-\mathrm{H}$ aliphalic vibrations peaking at $2845 \mathrm{~cm}^{-1}$ and $\mathrm{C}-\mathrm{H}$ aromatic vibrations peaking at $3054 \mathrm{~cm}^{-1}$ while PMMA beads lack C-H aromatic vibrations, hence PS beads remained visible on both images but the PMMA beads disappeared when the Raman shift was switched from $2845 \mathrm{~cm}^{-1}$ to $3035 \mathrm{~cm}^{-1}$. This wavelength switching was realised by manually translating the OPO crystal mount which could be performed at sub-second rates. This switching time could be reduced further by locating the crystal and mount on a fully motorized stage. It should be noted that the tuning of the crystal grating did not misalign the OPO cavity and that the stability of the OPO was not affected by manually repositioning the crystal mount.

\section{Discussion}

We have demonstrated a simple, efficient, inexpensive and flexible modular OPO laser system with nearly perfect beam quality, broad tunablity and excellent stability for CARS microscopy. CARS imaging was successfully performed using the single Ti:Sapphire laser platform. The CARS microscope provided very good spatial resolution images and fast frequency tuning capability. Furthermore, due to the spectrally versatile outputs of the laser system, confocal, two-photon and CARS 
imaging can be simultaneously performed on the same microscope platform, thus providing a one-stop solution for the wider life sciences community. 


\section{Acknowledgements}

We would like to thank Dr. Andreas Volkmer of the University of Stuttgart for discussions regarding laser stability in CARS microspectroscopy. This project is funded by the Biotechnology and Biological Sciences Research Council (BBSRC). 


\section{References}

Boyd GD, Kleinman DA. 1968. Parametric interation of focused Gaussian light beams. J. Appl. Phys. 39: 3597-3639.

Cheng JX, Volkmer A, Book LD, Xie XS. 2001. An Epi-Detected Coherent Anti-Stokes Raman Scattering (E-CARS) Microscope with High Spectral Resolution and High Sensitivity. J. Phys. Chem. B. 105: 1278.

Chen JX, Jia YK, Zheng GF, Xie XS. 2002a. Laser-Scanning Coherent Anti-Stokes Raman Scattering Microscopy and Applications to Cell Biology. Biophys. J. 83: 502-509.

Cheng JX, Volkmer A, Xie XS. 2002b. Theoretical and experimental characterization of coherent anti-Stokes Raman scattering microscopy. J. Opt. Soc. Am. B. 19: 1363-1375.

Chen JX, Xie XS. 2004. Coherent Anti-Stokes Raman Scattering Microscopy: Instrumentation, Theory, and Applications. J. Phys. Chem. B. 108: 827-840.

Cheung EC, Liu JM. 1990. Theory of a synchronously pumped optical parametric oscillator in steady-state operation. J. Opt. Soc. Am. B. 7: 1385-1401.

Connor MVO, Watson MA, Shepherd DP, Hanna DC. 2004. Use of a birefringent filter for tuning a synchronously pumped optical parametric oscillator. Appl. Phys. B. 79: $15-23$.

Evans CL, Potma EO, Puorishaag M, Cote D, Lin CP, Xie XS. 2005. Chemical imaging of tissue in vivo with video-rate coherent anti-Stokes Raman scattering microscopy. Proc. Natl. Acad. Sci. 102: 16807-16812.

Evans CL, Xie XS. 2008. Coherent anti-Stokes Raman scattering microscopy: chemical imaging for biology and medicine. Annu. Rev. Anal. Chem. 1: 883-909.

Fejer MM, Magel GA, Jundt DH, Byer RL. 1992. Quasi-phase-matching second harmonic generation: tuning and tolerances. IEEE J. Quan. Electron. 28: 2631-2654.

Ganikhanov F, Carrasco S, Xie XS, Katz M, Seitz W, Kopf D. 2006. Broadly tunable dual-wavelength light source for coherent anti-Stokes Raman scattering microscopy. Opt. Lett. 31: 1292-1294.

Guha S. 1998. Focusing dependence of the efficiency of a singly resonant optical parametric oscialltor. Appl. Phys. B. 66: 663-675.

Hache A, Allan GR, Driel HMV. 1995. Effects of cavity detuning on the pulse characteristics of a femtosecond synchronously pumped optical parametric oscillator. J. Opt. Soc. Am. B. 12: 2209-2213.

Hashimoto M, Araki T, Kawata S. 2000. Molecular vibration imaging in the fingerprint region by use of coherent anti-Stokes Raman scattering microscopy with a collinear configuration. Opt. Lett. 25: 1768-1770.

Jones DJ, Potma EO, Cheng JX, Burfeindt B, Pang Y, Ye J, Xie XS. 2002. Synchronization of two passively mode-locked, picosecond lasers within $20 \mathrm{fs}$ for coherent anti-Stokes Raman scattering microscopy. Rev. Sci. Instrum. 73: 2843-2848. Keatings SR, Zhang W, McConnell G. 2008. Characterization of microscope objective lenses from 1400 to $1650 \mathrm{nmto}$ evaluate performance for long-wavelength nonlinear microscopy applications. Microsc. Res. Tech. 71: 517-520. 
Levenson MD, Kano SS. 1988. Introduction to Nonlinear Laser Spectroscopy. San Diego: Academic. 148 p.

Moger J, Johnston BD, Tyler CR. 2008. Imaging metal oxide nanoparticles in biological structures with CARS microscopy. Opt. Exp. 16: 3408-3419.

Myers LE, Eckardt RC, Fejer MM, Byer RL, Bosenberg WR, Pierce JW. 1995. Quasi-phase-matched optical parametric oscillators in bulk periodically poled $\mathrm{LiNbO}_{3}$. J. Opt. Soc. Am. B. 12: 2102-2116.

Onorato RM, Muraki N, Knutsen KP, Saykally RJ. 2007. Chirped coherent anti-Stokes Raman scattering as a high-spectral- and spatial-resolution microscopy. Opt. Lett. 32: 2858-2860.

Potma EO, Jones DJ, Cheng JX, Xie XS, Ye J. 2002. High-sensitivity coherent anti-Stokes Raman scattering microscopy with two tightly synchronized picosecond lasers. Opt. Lett. 27: 1168-1170.

Shcherbo D, Murphy CS, Ermakova GV, Solovieva EA, Chepurnykh TV, Shcheglov AS, Verkhusha VV, Pletnev VZ, Hazelwood KL, Roche PM, Lukyanov S, Zaraisky AG, Davidson MW, Chudakov DM. 2009. Far-red fluorescent tags for protein imaging in living tissues. Biochem. J. 418: 567-574.

Volkmer A. 2005. Vibrational imaging and microspectroscopies based on coherent anti-Stokes Raman scattering microscopy. J. Phys. D: Appl. Phys. 38: R59-R81.

Zumbusch A, Holtom GR, Xie XS. 1999. Three-dimentional vibrational imaging by coherent anti-Stokes Raman scattering. Phys. Rev. Lett. 82: 4142-4145. 
Table

\begin{tabular}{|c|c|c|}
\hline Grating Period $(\Lambda)$ & Pump Range & Raman Range \\
\hline $19.8 \mu \mathrm{m}$ & $755 \mathrm{~nm}-786 \mathrm{~nm}$ & $2375 \mathrm{~cm}^{-1}$ to $4389 \mathrm{~cm}^{-1}$ \\
\hline $19.6 \mu \mathrm{m}$ & $750 \mathrm{~nm}-781 \mathrm{~nm}$ & $2463 \mathrm{~cm}^{-1}$ to $4470 \mathrm{~cm}^{-1}$ \\
\hline $19.4 \mu \mathrm{m}$ & $745 \mathrm{~nm}-776 \mathrm{~nm}$ & $2553 \mathrm{~cm}^{-1}$ to $4553 \mathrm{~cm}^{-1}$ \\
\hline $19.2 \mu \mathrm{m}$ & $740 \mathrm{~nm}-771 \mathrm{~nm}$ & $2643 \mathrm{~cm}^{-1}$ to $4636 \mathrm{~cm}^{-1}$ \\
\hline $19.0 \mu \mathrm{m}$ & $735 \mathrm{~nm}-766 \mathrm{~nm}$ & $2735 \mathrm{~cm}^{-1}$ to $4721 \mathrm{~cm}^{-1}$ \\
\hline $18.8 \mu \mathrm{m}$ & $730 \mathrm{~nm}-761 \mathrm{~nm}$ & $2829 \mathrm{~cm}^{-1}$ to $4807 \mathrm{~cm}^{-1}$ \\
\hline $18.6 \mu \mathrm{m}$ & $725 \mathrm{~nm}-756 \mathrm{~nm}$ & $2923 \mathrm{~cm}^{-1}$ to $4894 \mathrm{~cm}^{-1}$ \\
\hline $18.4 \mu \mathrm{m}$ & $720 \mathrm{~nm}-751 \mathrm{~nm}$ & $3019 \mathrm{~cm}^{-1}$ to $4982 \mathrm{~cm}^{-1}$ \\
\hline $18.2 \mu \mathrm{m}$ & $715 \mathrm{~nm}-746 \mathrm{~nm}$ & $3116 \mathrm{~cm}^{-1}$ to $5071 \mathrm{~cm}^{-1}$ \\
\hline $18.0 \mu \mathrm{m}$ & $710 \mathrm{~nm}-741 \mathrm{~nm}$ & $3215 \mathrm{~cm}^{-1}$ to $5162 \mathrm{~cm}^{-1}$ \\
\hline $17.8 \mu \mathrm{m}$ & $705 \mathrm{~nm}-736 \mathrm{~nm}$ & $3315 \mathrm{~cm}^{-1}$ to $5253 \mathrm{~cm}^{-1}$ \\
\hline $17.6 \mu \mathrm{m}$ & $700 \mathrm{~nm}-730 \mathrm{~nm}$ & $3416 \mathrm{~cm}^{-1}$ to $5365 \mathrm{~cm}^{-1}$ \\
\hline
\end{tabular}

Table 1, Pump tuning range for each crystal grating period at a fixed temperature of $393 \mathrm{~K}$. 


\section{Figure Captions}

Figure 1, Schematic setup of the CARS microscope. FI, Faraday isolator; HW, half wave plate; PL, polarizer; M1-M10, highly reflective mirrors for near infrared region; $\mathrm{CM} 1, \mathrm{CM}$ 2, OPO cavity concave mirrors $\left(\mathrm{CaF}_{2}\right.$ substrates) with radius of curvature of $100 \mathrm{~mm}$; both are highly reflective (HR) for signal beam and transparent for idler beam; CM1 has $>85 \%$ transmission for pump beam wavelength; PPLN, periodically poled lithium niobate crystal for parametric generation; OC, $40 \%$ reflectivity output coupling mirror for ps OPO and $50 \%$ reflectivity output coupling mirror for fs OPO; f1-f7, plano-concave lenses; NDW1-2, neutral density wheels; DM, dichroic mirror for combining the pump and the Stokes beams; FM, flip mirror; ADP, sum-frequency mixing crystal; BP1 and BP2, bandpass filters (Semrock FF01-769nm/41-25 and Chroma HQ430/50m-2p); PMT, photomultiplier tube.

Figure 2, OPO signal output power as a function of incident pump power; the inset shows the amplitude stability measurements for both the Ti:Sapphire laser and the OPO over 33 minutes.

Figure 3, Measured OPO wavelength at different temperatures and grating periods (data points); theoretical grating tuning curves at different temperatures (solid lines).

Figure 4, The autocorrelation trace of the ps-OPO pulse at a negative cavity detuning length of $40 \mu \mathrm{m}$ (a) and its corresponding spectrum (b); the ps-OPO output power and the wavelength as a function of the cavity length detuning in the range of $-100 \mu \mathrm{m}$ to $+60 \mu \mathrm{m}$ (c) and time-bandwidth (TBW) product of the laser pulse versus cavity length detuning $(\mathrm{d})$.

Figure 5, The autocorrelation width (FWHM) and the wavelength as a function of the cavity length mismatch in the range of $-60 \mu \mathrm{m}$ to $+60 \mu \mathrm{m}$ (a) and the time-bandwidth (TBW) product of the laser pulse versus cavity length detuning (b).

Figure 6, The measured CARS spectrum (in circles) and the corresponding spontaneous Raman spectrum of $1 \mu \mathrm{m}$ polystyrene beads (a); CARS image of $1 \mu \mathrm{m}$ beads at Raman shift of $3035 \mathrm{~cm}^{-1}$, the image size was $262 \times 165$ (pixels) ${ }^{2}$, the scale bar represents $5 \mu \mathrm{m}$ (b); image in the xz plane of a single bead, image size was $95 \mathrm{x}$ 47 (pixels) $^{2}$ (c); the intensity profile of the bead in xy plane (d); and the intensity profile in $x z$ plane $(\mathrm{e})$.

Figure 7, CARS images of the mixture of $10 \mu \mathrm{m}$ polystyrene beads and $1.4 \mu \mathrm{m}$ PMMA beads at Raman shifts of $2848 \mathrm{~cm}^{-1}$ (a) and $3035 \mathrm{~cm}^{-1}$ (b); the image size was $512 \times 512$ (pixels) $^{2}$ and both images were averaged over 5 frames with a frame capture rate of $1.68 \mathrm{~s}$. 


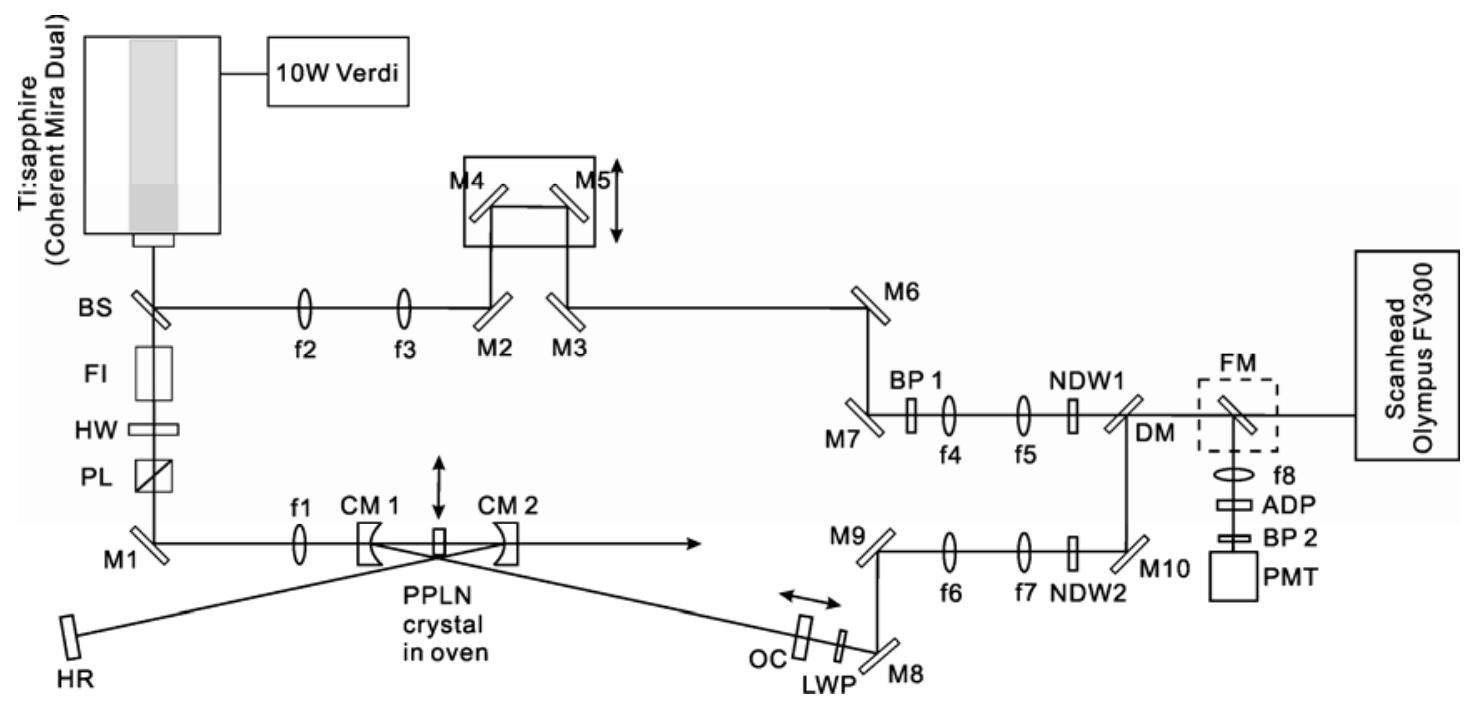




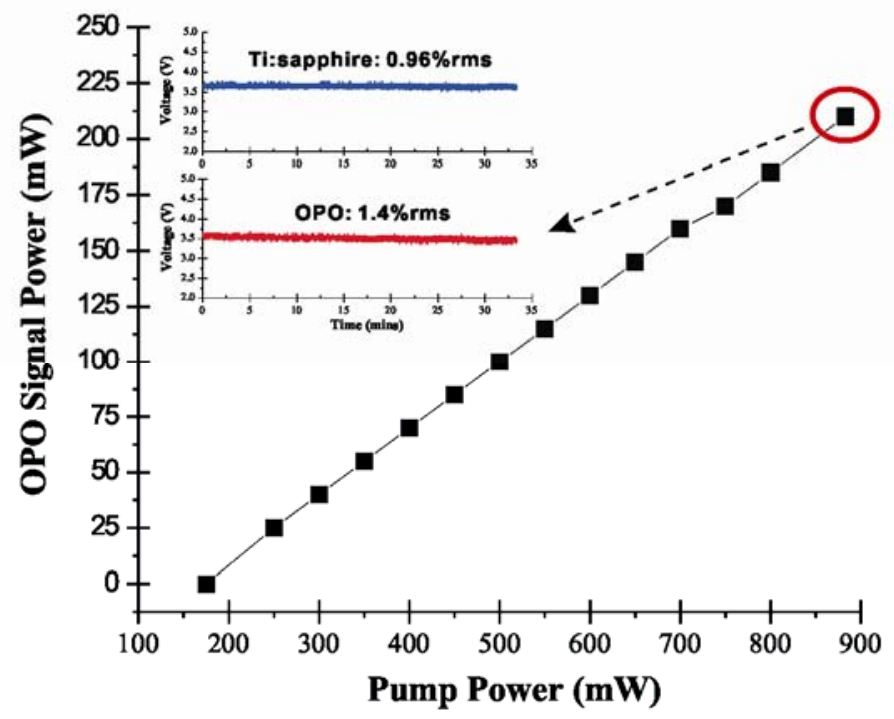




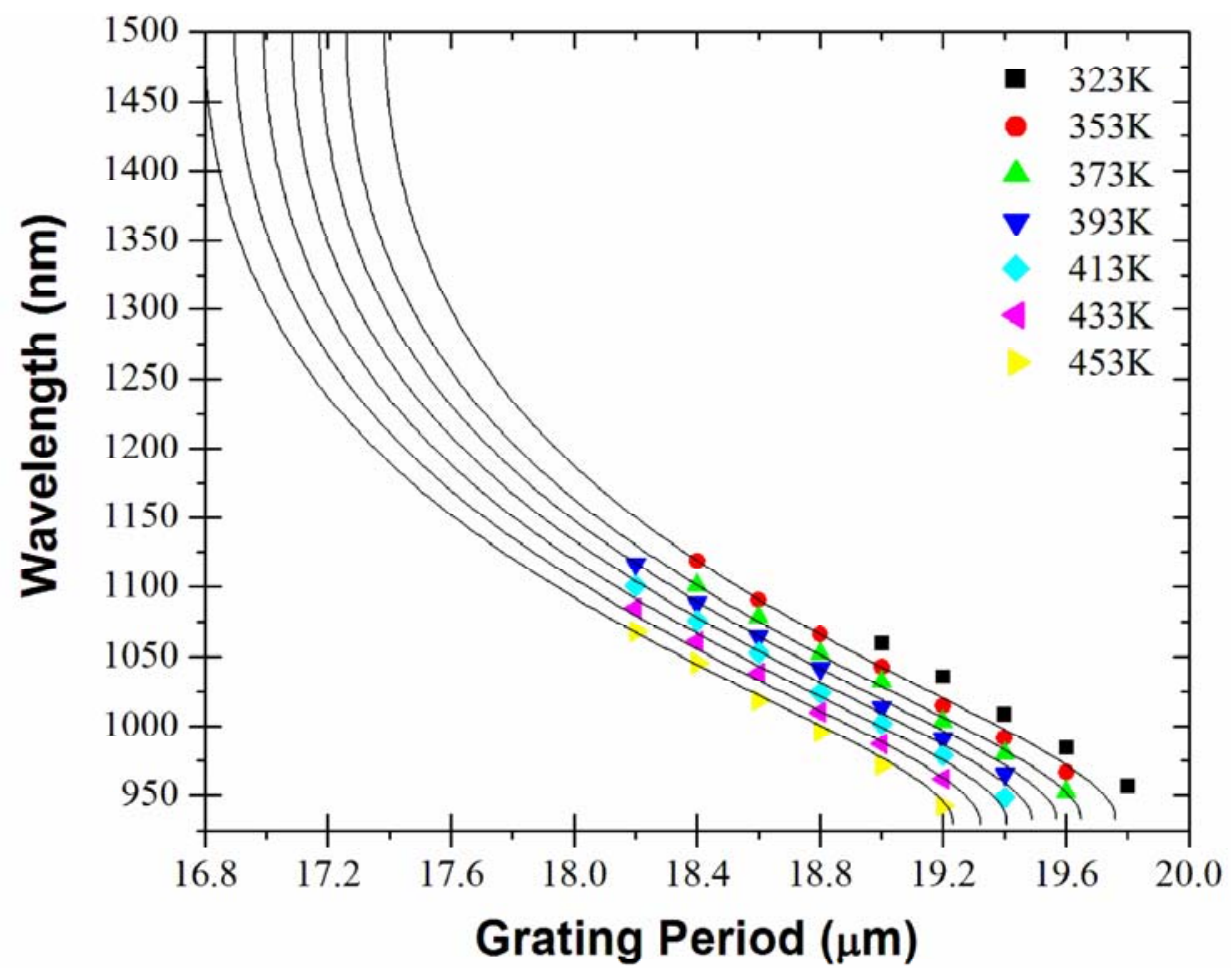




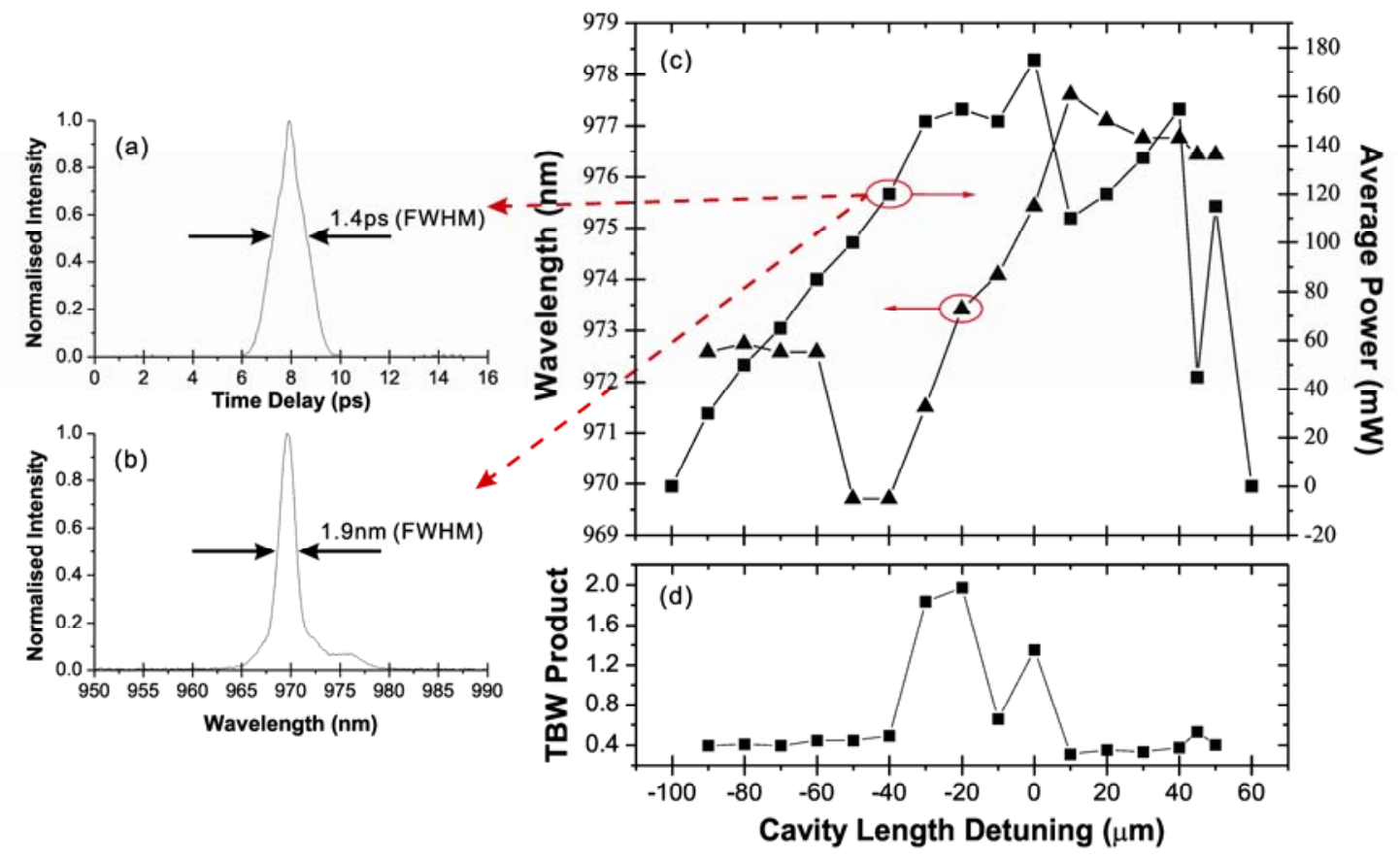




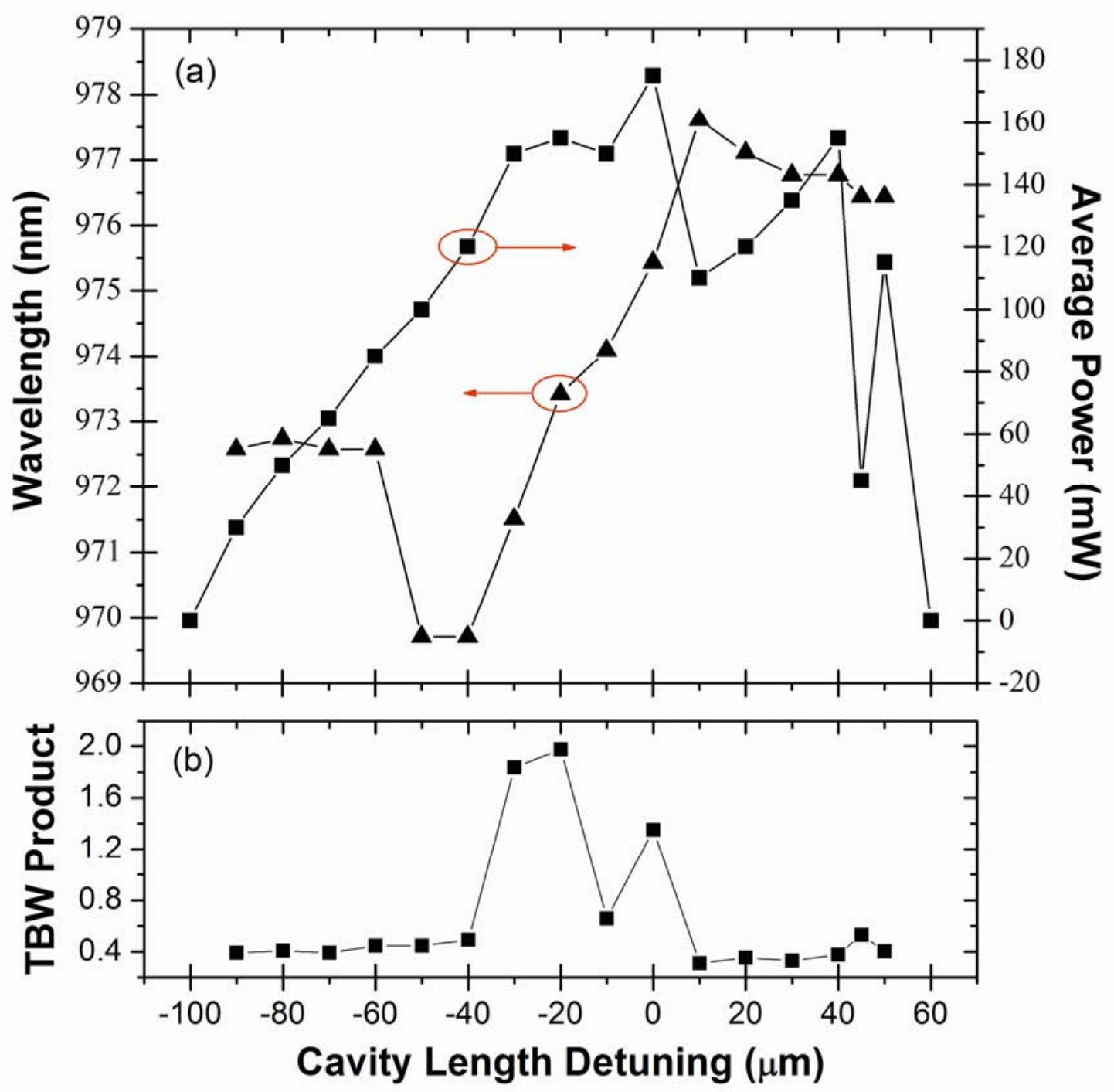



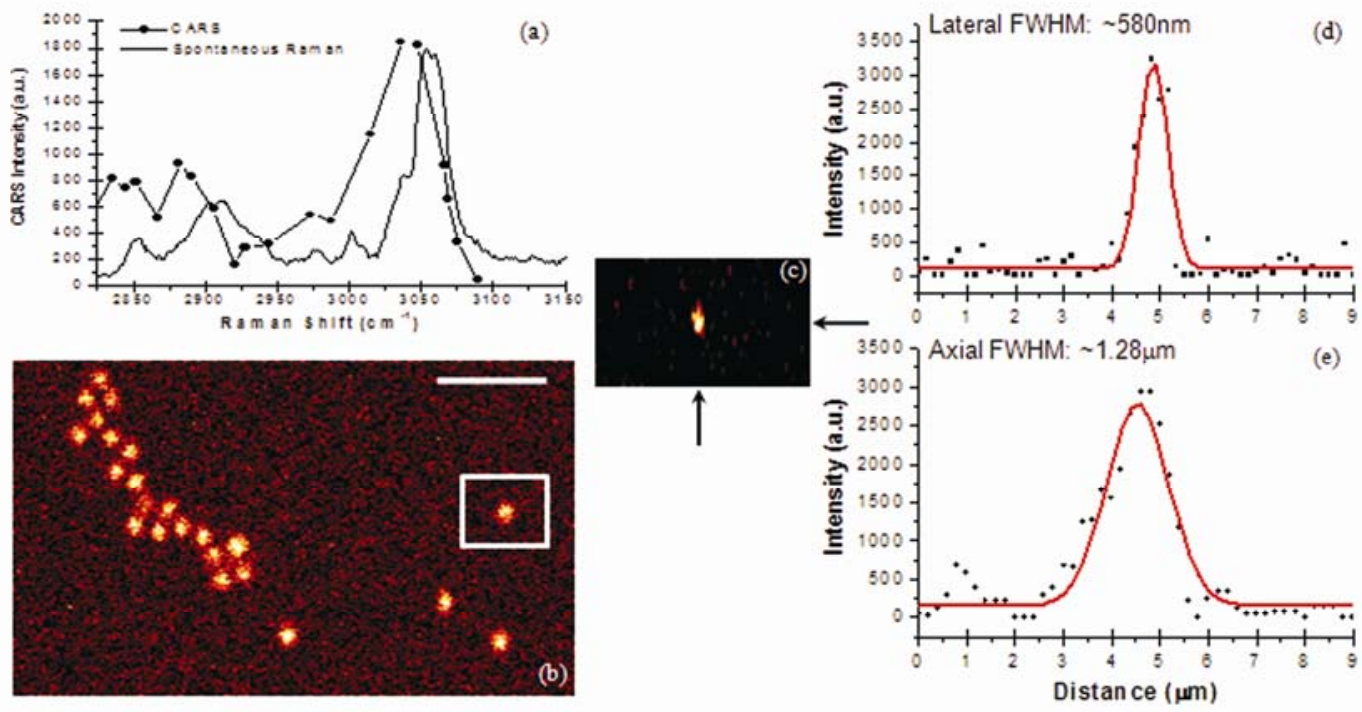


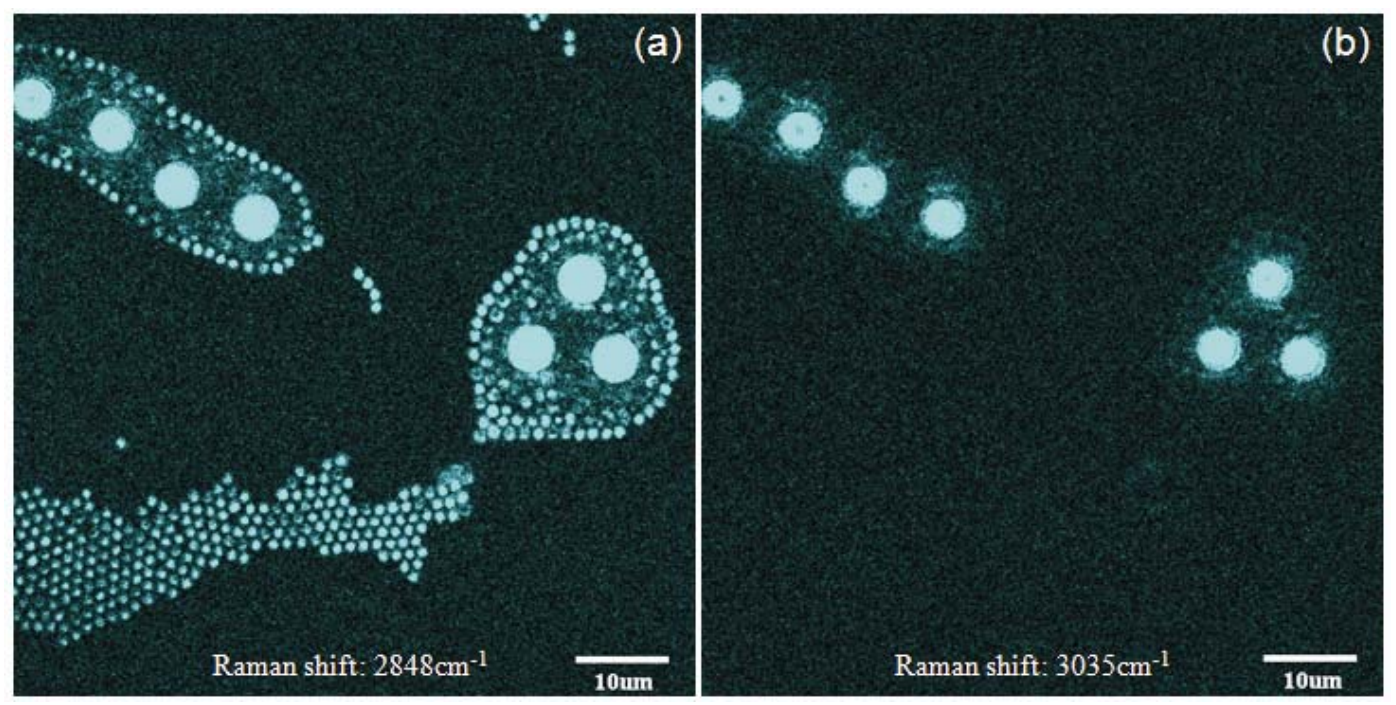

